\title{
COVID-19 Infection: Impact on Hair
}

\author{
Sandeep Suresh Sattur ${ }^{1}$ Indu Sandeep Sattur ${ }^{1}$ \\ ${ }^{1}$ Hairrevive-Centre for Hair Restoration \& Skin Rejuvenation, \\ Santacruz West, Mumbai, Maharashtra, India \\ Indian J Plast Surg 2021;54:521-526.
}

\begin{abstract}
Address for correspondence Sandeep Suresh Sattur, M Ch, Hairrevive-Centre for Hair Restoration \& Skin Rejuvenation, 103, Options Commercial Centre, Dr. Vasant Awsare Marg, Milan Subway Road, Santacruz West, Mumbai, Maharashtra, 400054, India (e-mail:drsattur@yahoo.com).
\end{abstract}

\begin{abstract}
Keywords

- hair fall

- COVID-19

- Hair follicle

With a practice dedicated to hair restoration and hair loss management, the authors have seen a large number of patients presenting with increased hair fall in the last 18 months. Hair fall after a stressfull event such as infections with febrile episodes is known. However, the latent period between the infective episode and hair fall has been shorter after COVID-19 infection than before. This review attempts to look at various pathways responsible for hair fall and if this differs from the conventional hair fall seen after any infection. It also tries to understand if the infection with the SARS-CoV-2 virus has a direct impact on the hair follicle leading to hair fall.
\end{abstract}

\section{Introduction}

Coronavirus disease 19 (COVID-19) is the third documented spill-over of an animal coronavirus to humans and can be regarded as a systemic viral illness based on its involvement in multiple major organ systems. ${ }^{1}$ This novel coronavirus $(\mathrm{CoV})$ is a positive-stranded RNA virus with a crown-like appearance caused by spike glycoproteins on the envelope designated as severe acute respiratory syndrome-related coronavirus-2 (SARS-CoV-2). ${ }^{1,2}$

The story that began in the city of Wuhan of the Hubei province of China has spread to most parts of the world. ${ }^{3}$ As per the World Health Organization (WHO), the cumulative number of cases reported globally is now over 190 million and the number of deaths exceeded 4 million as of July $2021 .{ }^{4}$

More than a year into the pandemic and we have learned to expect the unexpected and are still uncovering different ways the SARS-COV2 virus is affecting humans. Moreover, since the start of the ongoing pandemic, the authors who have a practice dedicated to hair restoration, have been approached by a large number of patients presenting with effluvium most of whom suffered COVID-19 infection but there were a few who did not have any history of infection.

published online

December 13, 2021
DOI https://doi.org/

10.1055/s-0041-1740289. ISSN 0970-0358.
Hair fall (telogen effluvium [TE]) after bacterial or viral or protozoal infections is not a new phenomenon and was also reported after the 1918 influenza epidemic. ${ }^{5-8}$ Literature review in terms of the impact of COVID-19 infection on the hair follicle reveals hair loss caused during and post-recovery, majorly manifesting as TE. ${ }^{8-12}$

This review attempts to understand if the mechanism of effluvium post-COVID-19 is similar to that seen with any other infections, as a response to stress created by febrile episodes or whether COVID-19 infection has a direct impact on hair follicles. Moreover, it tries to identify if the hair fall is due in addition to a combination of various factors such as cytokines, medications used during the treatment (antivirals, antibiotics, steroids, anticoagulants, etc.), the psychological impact of the infection, and local restrictions/ quarantine and ambient stress of the pandemic.

\section{Understanding Pathogenesis of COVID-19 Infection}

The SARS-CoV-2 virus spreads mainly via respiratory droplet transmission and through the air (airborne transmission) or fomites. ${ }^{3}$ Once the virus gains entry into the host, the spike receptor-binding domain (RBD) allows the binding to the
(C) 2021. Association of Plastic Surgeons of India. All rights reserved. This is an open access article published by Thieme under the terms of the Creative Commons Attribution-NonDerivative-NonCommercial-License, permitting copying and reproduction so long as the original work is given appropriate credit. Contents may not be used for commercial purposes, or adapted, remixed, transformed or built upon. (https://creativecommons.org/ licenses/by-nc-nd/4.0/)

Thieme Medical and Scientific Publishers Pvt. Ltd., A-12, 2nd Floor, Sector 2, Noida-201301 UP, India 
angiotensin-converting enzyme 2 (ACE2) receptor in the lungs and other tissues. Primarily, it impacts the lungs but affects most of the organs including the skin. ${ }^{2}$ Viral replication results in direct tissue damage in the early phase which is followed by a more severe late phase in some patients. The more severe late stage is marked by an immune response by the infected host cells with the recruitment of $\mathrm{T}$ lymphocytes, monocytes, and neutrophil recruitment. Release of cytokines such as tumor necrosis factor- $\alpha$ (TNF $\alpha)$, granulocyte-macrophage colony-stimulating factor (GM-CSF), interleukin-1 (IL-1), interleukin-6 (IL-6), IL-1 $\beta$, IL-8, IL-12 and interferon (IFN)- $\gamma^{3}$ or the so-called cytokine storm is the cause of multi-organ injury. 2,3

\section{Hair and Skin Involvement by SARS-CoV-2 Virus}

The significance of cutaneous involvement in COVID-19 was not noticed in the early phases of the pandemic but was observed much later and was crucial as it was a presenting complaint in some patients. ${ }^{10-14}$ Though the pathophysiology of the rash or exanthema ${ }^{14}$ still eludes us, the virus is thought to enter cutaneous tissue through the blood vessels as the endothelium is known to express ACE-2 initiating an inflammatory response. ${ }^{15}$ Physiological stress and the circulating cytokines created by viral infection impact not only the keratinocytes in the skin but also the rapidly dividing dermal papilla matrix cells. ${ }^{16}$

\section{Impact on Hair Follicles}

The effluvium caused post the infection with SARS COV2 could be explained by the physiologic response of the follicles to the stress of the infection. After a fever-causing infection, the effluvium usually starts 3 to 4 months after the inciting infection. The cytokines released due to the infection/fever, prematurely push the follicles into catagen and then into telogen. As the duration of telogen is $\sim 3$ to 4 months, the shedding usually starts at this time. Many of the studies as well as the personal experience of the authors have found that the interval between the infection and onset of the effluvium is shorter than that seen with other febrile conditions, starting as early as 4 weeks after the infection, and sometimes it has even been reported during the infection. ${ }^{5,9,17}$ Due to these findings, it raises the suspicion if the SARS-CoV-2 causes direct injury to the hair follicle. The authors have tried to put forward potential mechanisms by which the SARS-CoV-2 virus may interact with the hair follicle directly or cause injury to it.

\section{Direct Follicular Injury}

It is known that for the SARS-CoV-2 virus to enter the host cells it needs to engage the ACE- 2 receptor (a zinc metallopeptidase), whereas TMPRSS2-a host cellular serine protease transmembrane protease serine-2 is needed to cleave the viral spike protein to facilitate virus fusion to the host cell membrane. ${ }^{18-20}$ Recent studies have shown that both these are expressed in the skin with ACE2 also found to be expressed in the basal layers of the hair follicle. Cells expressing these proteins could be potential targets of SARS-CoV-2 and possible cellular damage thereby providing an avenue for the virus to injure the hair follicle. ${ }^{9}$ But with limited studies assessing follicular damage, it is inconclusive to deduce if the skin lesions are a site of viral replication or merely a cutaneous response to systemic infection. ${ }^{18-21}$

\section{Antibody-Dependent Enhancement}

Apart from ACE2 receptors, another possible pathway that could allow the virus access to the follicle is the antibodydependent enhancement (ADE). ${ }^{22,23}$ Theoretically this could occur if the person is infected with a different strain of COVID-19 or could be seen after hyperimmune globulin therapy or even after vaccination. ${ }^{24}$

Normally virus-specific antibodies are able to neutralize the virus and prevent the spread of the infection. Sometimes, however, these antibodies are sub-neutralizing in nature and enhance the entry of the virus and or help in the replication of viruses, leading to the phenomenon of ADE. Antibodies or Immunoglobulins have two Fragment antigen-binding or "Fab" sites and one Fragment crystallizable or "Fc" region. The Fc region has sites for binding to Fc receptors on effector cells and normally its major function is to clear the antigenantibody complexes via receptor-mediated endocytosis. ${ }^{25}$ ADE works through interaction with Fc and/or complement receptors. This mechanism is well known in dengue infections, but it is also seen with coronaviruses such as SARSCoV-1 and MERS and it could exist for COVID-19 as well. ${ }^{22,24}$ Studies have demonstrated that the Fc receptors are functionally expressed in human keratinocytes and as well as human hair follicle dermal papilla cells (HFDPCs). ${ }^{26}$ With limited literature support it is too early to accept or reject the hypothesis and clinical relevance of ADE in SARS-CoV-2 but keeping in mind the above findings, ADE induced hair follicle damage in COVID-19 cannot be ruled out completely.

\section{Apoptosis}

Programmed cell death or apoptosis is an evolutionarily conserved mechanism to combat infection or remove damaged or infected cells - a strategy of the host to restrict the spread of the virus. ${ }^{27}$ It is seen in many viral infections such as dengue and Japanese encephalitis virus. In-vitro studies using HFDPCs and dengue virus have shown activation of caspase cascade, reduced level of BMP-4 (bone morphogenetic protein4) along with downregulation of noggin (NOG), all of which indicate dysregulation of hair growth in the HFDPCs. ${ }^{26}$ Once the virus gains access to the hair follicle, this could be another pathway that can work toward causing premature induction of catagen and further on telogen leading to increased hair fall. Whether this mechanism is operational in COVID-19 patients is not known presently due to limited published data on the structural impact on the hair follicle and as more studies are conducted the picture may get clearer.

\section{Cytokines}

COVID-19 infection elicits a strong antiviral response in form of the release of cytokines such as TNF $\alpha$, GM-CSF, IL-1, IL-6, 
IL-1 $\beta$, IL-8, IL-12, and interferon (IFN)- $\gamma^{3}$ Many of these factors impact the rapidly dividing keratinocytes and dermal papilla cells. TNF, IL-6, and interferon all are known to be inhibitory to hair follicle growth in-vitro. ${ }^{22,28,29}$ IL4 which is increased in the elderly is known to cause keratinocyte apoptosis. $^{22}$ In a longitudinal study of COVID-19 survivors in Wuhan, 534 patients were followed up three months postdischarge, alopecia was observed in 154 patients. Most of these patients complained of alopecia after discharge (classic telogen effluvium) but almost one-third of these patients had alopecia during hospitalization. ${ }^{5} \mathrm{~A}$ similar case report of effluvium during hospitalization with COVID-19 was also reported. ${ }^{17}$ Any event that suddenly disrupts the mitotic or metabolic activity of the hair follicle is known to cause the shedding of hairs in the growing phase (anagen effluvium). Most commonly it is seen after chemotherapy/radiotherapy but could also be due to sudden exposure to pro-inflammatory factors and cytokines. ${ }^{17,30}$ Probably the intensity of the inflammatory response occurring in a short time frame, impacts the onset of hair fall in these patients, and in such cases, there may be no latent period between the actual insult to the hair follicle and the onset of hair fall. It would be important to consider here that underlying nutritional deficiencies may contribute or exaggerate the effluvium post COVID.

\section{Microthrombi}

The virus is thought to reach the skin through blood vessels facilitated by the presence of ACE2 receptors in the endothelium. The ensuing inflammatory reaction to the viral invasion and subsequent immune response sets in motion a cascade of events leading to cytokine release by the $\mathrm{CD} 4^{+}$ $\mathrm{T}$ helper cells and recruitment of eosinophils, $\mathrm{CD}^{+}$cytotoxic T cells, B cells, and natural killer (NK) cells finally culminating in lymphocytic thrombophilic arteritis. ${ }^{15,31}$ Histopathological evaluation of cutaneous lesions in COVID-19 has revealed intense perivascular lymphocytic and plasmacytic infiltrate with extravasation of red blood cells and intraluminal thrombi. ${ }^{15,32}$ These microvascular thrombi could theoretically play a role in creating local cutaneous tissue hypoxia thereby affecting the hair follicles. This could be another way the SARS-COV2 could impact the hair follicles. More in vitro studies and PCR investigations on skin biopsies would be needed along with histopathological analyses to include the status of hair follicles.

\section{Therapies Used to Treat COVID-19}

Patients hospitalized for COVID-19 are exposed to an array of drugs including antibacterials, antivirals, anticoagulants, corticosteroids, and immunomodulators. ${ }^{3}$ Literature shows that anticoagulants and antivirals and even antibiotics may cause hair fall in some cases., ${ }^{3,8,33}$ of these anti-coagulants may play a major role. Anticoagulation could be therapeutic or prophylactic and is indicated in hospitalized patients with almost $80 \%$ of the patients hospitalized for COVID-19 receiving anticoagulants in some form or the other. ${ }^{34}$ The exact cause of hair fall with the use of anticoagulants is not known as most studies point toward telogen effluvium. Theories ranging from focal degeneration of collagen bundles in the vicinity of vessels of the follicular connective sheath or direct injury to connective tissue papilla have been put forth but presently there is no consensus on this matter but it is agreed that anticoagulants can cause hair fall. ${ }^{33}$ The anticoagulants implicated are low molecular weight heparins and direct oral anti-coagulants like rivaroxaban and dabigatran and apixaban. ${ }^{33}$ More importantly a combination of the severity of disease necessitating hospitalization and use of anticoagulants could be another possible mechanism to cause insult to the hair follicle.

\section{Psychological Stress}

COVID-19 pandemic has impacted people psychologically at multiple levels. Whether it is the general population or the health care workers or the vulnerable population or those affected by the infection-everyone has been impacted at some level. ${ }^{35,36}$ As part of non-pharmacological control of the spread of COVID-19, various governmental and medical organizations recommended social/physical distancing, wearing a mask at all times in public places, maintaining personal hygiene and lockdowns with isolation of COVID-19 positive and suspected COVID-19 patients, quarantine of exposed individuals, travel limitations, closure of educational institutions and workplaces and prohibition of mass gatherings. Though these measures helped in controlling the spread of the disease they also caused significant psychological impact and quarantine and isolation have been found to be the most associated with severe psychological stress. ${ }^{37,38}$ During quarantine or isolation or hospitalization the patient is separated from family/friends, there is the uncertainty of disease progression and status, reduced communication with healthcare professionals and boredom significantly affects the patient's psyche. Constant updates about the number of new cases, deaths, etc. due to the pandemic on social media are also additional stressors to the patient in quarantine or isolation. A multicentric, multinational study has shown that as the duration of quarantine increased, the quantum of perceived stress also increased proportionally. ${ }^{38}$

Also, the restrictions imposed to prevent the spread of the pandemic, have led to social isolation which has triggered feelings of anxiety and depression in people and this itself could also be a cause for increased hair fall. ${ }^{9}$

Literature is replete with reports of hair loss associated with psycho-emotional stress. ${ }^{39-42}$ Stress can impact hair loss as a primary factor or inducer of effluvium or it can be a contributing factor in face of an existing medical problem which in this case is COVID-19 infection. Stress can also be caused by hair fall in which case the stress could work on a secondary basis to aggravate the hair fall. ${ }^{42}$

It is important to understand how stress affects the hair follicle. At a biological level, we (as in all living organisms) have an inbuilt system that responds by adapting and protecting us from exogenous and endogenous stressors (including psychological and social). ${ }^{39}$ The classic stress response involves the activation of the sympatheticAdreno-Medullar (SAM) axis, the Hypothalamus-Pituitary- 
Adrenal (HPA) axis, and the immune system. However, if the stress is severe or persists or is repetitive, the adaptive response harms the organism. ${ }^{40}$ The quick response involves increased secretion or epinephrine and norepinephrine from the adrenal medulla and helps the organism prepare for the fight or flight action. The relatively slower response involves activation of the hypothalamic-pituitary-adrenal (HPA) axis with the release of corticotropin-releasing hormone (CRH) and activation of pituitary $\mathrm{CRH}$ receptors (CRH-R) leading to the production and release of proopiomelanocortin-derived peptides and adrenal hormones. ${ }^{39,40}$

Apart from the above-mentioned SAM axis and HPA axis, there exists a brain-hair follicle axis that is responsible for hair growth-limiting effects of psycho-emotional stress. ${ }^{42}$ Experimental animal studies have revealed that perceived stress can trigger neuroendocrine immune responses which cause neurogenic inflammation and promote apoptosis in hair follicles. Like in mouse studies it has been postulated that stress activates neuroendocrine-immune circuits in humans as well. ${ }^{41}$ These circuits are thought to be present at self/environment interface like the skin and which interact with mast cells activating them in periods of psycho-emotional stress. Psychological stress is known to significantly increase the stimulated production of pro-inflammatory cytokines such as TNF $\alpha$, IL-6, IFN- $\gamma$ by the activation of the mast cells. ${ }^{41}$ These cytokines released in response to intense stress are known to drive epithelial and mesenchymal cells into apoptosis causing premature termination of hair growth. Peters et al have reasonably concluded that stress can lead to temporary hair fall in healthy individuals by correlating increased levels of salivary cortisol levels and blood levels of cytokines to hair fall in healthy individuals exposed to psychological stress. ${ }^{41}$

Telogen effluvium by itself could be a source of stress and may further propagate hair fall in the affected patients. ${ }^{42}$ This could be another source of hair fall observed in patients post recovery from COVID-19.

COVID-19 has also been associated with rapidly progressive forms of alopecia areata. It has been known to aggravate existing alopecia areata in a patient after recovering from COVID-19 that progressed to universalis. Stress caused by the pandemic and lockdown measures also appears to have triggered alopecia areata in patients who were antibody (IgG) negative for SARS-CoV-2 but did have the stress of isolation. $^{43}$

Another issue of hair fall seen during the pandemic by the authors is a perceived increase in hair fall or pseudoeffluvium. As we know it is fairly normal to lose 100 to 150 hairs on a daily basis which is not noticed. ${ }^{43}$ But since restrictions were levied on the population to prevent the spread of COVID-19, most people were homebound and that is when people noticed an increase in hair fall as they would see a lot of hair around the house and panic at the sight of the shed hair around the house without actually having true effluvium. In most of these patients, the assessment revealed a negative hair pull and normal trichoscopy. Most of the patients responded to reassurance and explanation given to them.

\section{Assessment and Management of Hair Fall Post-COVID-19 Infection}

The most common presenting complaint is a sudden onset of increased hair fall apparently without any reason as it starts when the patient has fully recovered from COVID-19. The functional type of telogen effluvium here is a synchronization of hair follicle growth cycles leading to immediate anagen release which occurs when stressors stimulate the follicles to leave anagen and enter telogen prematurely, resulting in increased hair fall after 2 to 3 months. ${ }^{44-46}$ On questioning there will be a history of being diagnosed with COVID-19 or febrile episodes in the recent past which many times the patient does not correlate with the hair fall. In some patients, there may be no such history. Additionally, there could be other triggers like any weight loss or nutrient deficiencies due to dietary restrictions, hypothyroidism, etc. Sometimes a unique complaint of "pain in the hair-trichodynia" is mentioned by the patient. It could take the form of an itchy feeling to the actual pain of needle prick when manipulating hair. It is thought to be associated with the severity of the hair fall but also may be seen in cases of co-existing pattern hair loss. ${ }^{46}$ However, no organic basis has been found for this symptom.

Examination of the patient's scalp may reveal diffuse thinning especially if the effluvium has been severe. One needs to lose close to $40 \%$ of hair for there to be a visual appreciation of hair loss. ${ }^{46}$ An important diagnostic test is the hair pull test. This test if properly done is pretty accurate in diagnosing telogen effluvium. It is important to know the patients' hair wash history to be able to interpret the test and one could look at the details of the test from the following references. ${ }^{44-47}$ Trichoscopy is probably one of the most important tools for assessing hair fall and hair loss. The findings seen in telogen effluvium are classic - reduced density and presence of empty ostia and absence of hair shaft diameter variability. ${ }^{48}$

An important differential diagnosis is diffuse alopecia areata or alopecia areata incognita. The clue here is there may be the presence of vellus hair. ${ }^{48}$

In terms of treatment, reassurance and patient education is the key to successfully reducing patient anxiety associated with hair fall. Most of these patients will need time and empathy from the treating doctor. There is no specific treatment other than countering the stressors. It is important to look for catagen inducing deficiencies like iron and protein deficiencies, thyroid function, and any drug such as $\beta$-blockers, retinoids, anticoagulants, etc. ${ }^{43}$

Minoxidil may be an option, ${ }^{43}$ especially if there is a coexisting/underlying pattern of hair loss. There have been reports of the use of topical steroid applications ${ }^{46}$ but the evidence is not very high. Getting the patient reassured, ensuring a nutritious high protein, high-calorie diet, and adequate sleep will go a long way in helping the patient deal with the hair fall.

\section{Conclusion}

The connection between cause and effect with respect to COVID-19 infection and hair fall is straightforward. The most 
obvious connection is the response of the hair follicle to increased biological stress created by the systemic infection and subsequent cytokine release leading to telogen effluvium. In typical telogen effluvium a latent period of 3 to 4 months exists between the trigger event and the onset of hair fall. In the authors' experience, patients who have recovered from COVID-19 had a much shorter latent period, as short as 4 weeks in some cases. Similar observations in some published studies have triggered the thought of a possible direct insult to the hair follicle caused by the SARS-CoV-2 virus. ${ }^{17,22,28}$ After exploring literature and rationalizing the possibilities, theoretically, there are ways for the virus to cause damage to the hair follicle but the present literature does not support this and more studies would be needed to conclusively prove that the SARS-CoV-2 causes direct injury to the follicle as it was shown in case of dengue virus. ${ }^{26}$ However, like all other telogen effluviums, almost all patients have a reversal of hair fall in a span of 2 to 3 months.

Another unique feature of the effluvium seen post-COVID19 is the significant overlay of psychoemotional stress. This is unlike any other infection, which leads to telogen effluvium because the ambient stress caused the pandemic itself and contributed to a large extent by the restriction measures taken to prevent the spread of the disease. Telogen effluvium would be one of the important aspects of long COVID, which will impact the quality of life of the affected person. With the pandemic still ongoing, the physicians dealing with COVID19 patients will have to be empathetic to this aspect of the infection and prepare the patients for the effluvium, and also reassure them that it is self-limiting and that regrowth is the norm.

In conclusion, a host of factors could be responsible for the severity and onset of hair fall in patients recovering from COVID-19 including the severity and duration of the COVID19 infection, treatment given, underlying nutritional deficiencies, patient's existing hair status, emotional lability, and psychological makeup. As a part of treatment reassurance and explanation that the hair fall is self-limiting and would reverse in $\sim 2$ to 3 months is a major stress buster for the patients and helps them to emotionally deal with the hair fall.

\section{Conflict of Interest}

None declared.

\section{References}

1 Coronaviridae Study Group of the International Committee on Taxonomy of Viruses. The species severe acute respiratory syndrome-related coronavirus: classifying $2019-\mathrm{nCoV}$ and naming it SARS-CoV-2. Nat Microbiol 2020;5(04):536-544. Doi: 10.1038/ s41564-020-0695-Z

2 Cascella M, Rajnik M, Aleem A, et al. Features, Evaluation, and Treatment of Coronavirus (COVID-19) [Updated 2021 Jul 17]. In: StatPearls [Internet]. Treasure Island (FL): StatPearls Publishing; 2021 Accessed November 17, 2021 from: https://www.ncbi.nlm. nih.gov/books/NBK554776/

3 Parasher A. COVID-19: Current understanding of its pathophysiology, clinical presentation and treatment. Postgrad Med J 2021; 97(1147):312-320
4 https://www.who.int/emergencies/diseases/novel-coronavirus2019/situation-reports

5 Xiong Q, Xu M, Li J, et al. Clinical sequelae of COVID-19 survivors in Wuhan, China: a single-centre longitudinal study. Clin Microbiol Infect 2021;27(01):89-95

6 Mieczkowska K, Deutsch A, Borok J, et al. Telogen effluvium: a sequela of COVID-19. Int J Dermatol 2021;60(01):122-124

7 Olds H, Liu J, Luk K, Lim HW, Ozog D, Rambhatla PV. Telogen effluvium associated with COVID-19 infection. Dermatol Ther (Heidelb) 2021;34(02):e14761

8 Rizzetto G, Diotallevi F, Campanati A, et al. Telogen effluvium related to post severe Sars-Cov-2 infection: clinical aspects and our management experience. Dermatol Ther (Heidelb) 2021;34 (01):e14547

9 Lv S, Wang L, Zou X, et al. A case of acute telogen effluvium after SARS-CoV-2 infection. Clin Cosmet Investig Dermatol 2021; 14:385-387

10 Recalcati S. Cutaneous manifestations in COVID-19: a first perspective. J Eur Acad Dermatol Venereol 2020;34(05):e212-e213

11 Fatemeh S, Bahareh H, et al. COVID-19 and skin manifestations: an overview of case reports/case series and meta-analysis of prevalence studies. JOURNAL Frontiers in Medicine 2020;7:651

12 Sachdeva M, Gianotti R, Shah M, et al. Cutaneous manifestations of COVID-19: Report of three cases and a review of literature. J Dermatol Sci 2020;98(02):75-81

13 Jia JL, Kamceva M, Rao SA, Linos E. Cutaneous manifestations of COVID-19: a preliminary review. J Am Acad Dermatol 2020;83 (02):687-690

14 Genovese G, Moltrasio C, Berti E, Marzano AV. Skin manifestations associated with COVID-19: current knowledge and future perspectives. Dermatology 2021;237(01):1-12

15 Deshmukh V, Motwani R, Kumar A, Kumari C, Raza K. Histopathological observations in COVID-19: a systematic review. J Clin Pathol 2021;74(02):76-83

16 Trüeb RM, Dutra Rezende H, Gavazzoni Dias MFR. What can the hair tell us about COVID-19? Exp Dermatol 2021;30(02):288-290

17 Shanshal M. COVID-19 related anagen effluvium. J Dermatolog Treat 2020:1-2. Doi: 10.1080/09546634.2020.1792400

18 Salamanna F, Maglio M, Landini MP, Fini M. Body localization of ACE-2: on the trail of the keyhole of SARS-CoV-2. Front Med (Lausanne) 2020;7:594495

19 Saponaro F, Rutigliano G, Sestito S, et al. ACE2 in the era of SARSCoV-2: controversies and novel perspectives. Front Mol Biosci 2020;7:588618

20 Radzikowska U, Ding M, Tan G, et al. Distribution of ACE2, CD147, CD26, and other SARS-CoV-2 associated molecules in tissues and immune cells in health and in asthma, COPD, obesity, hypertension, and COVID-19 risk factors. Allergy 2020;75(11):2829-2845

21 Hamming I, Timens W, Bulthuis ML, Lely AT, Navis G, van Goor H. Tissue distribution of ACE2 protein, the functional receptor for SARS coronavirus. A first step in understanding SARS pathogenesis. J Pathol 2004;203(02):631-637

22 Rossi A, Magri F, Sernicola A, et al. Telogen effluvium after SARSCoV-2 infection: a series of cases and possible pathogenetic mechanisms. Skin Appendage Disord 2021;21:1-5

23 Tirado SM, Yoon KJ. Antibody-dependent enhancement of virus infection and disease. Viral Immunol 2003;16(01):69-86

24 Ulrich H, Pillat MM, Tárnok A. Dengue fever, COVID-19 (SARS$\mathrm{CoV}-2$ ), and antibody-dependent enhancement (ADE): a perspective. Cytometry A 2020;97(07):662-667

25 Hajela K. Structure and function of Fc receptors. Biochem Educ 1991;19(02):50-57 ISSN 0307-4412

26 Wei KC, Huang MS, Tsung-Hsien C. Dengue VIPHHFDPC. Front Cell Infect Microbiol 2018;8:268

27 Okamoto T, Suzuki T, Kusakabe S, et al. Regulation of apoptosis during flavivirus infection. Viruses 2017;9(09):243

28 Abrantes TF, Artounian KA, Falsey R, et al. Time of onset and duration of post-COVID-19 acute telogen effluvium. J Am Acad 
Dermatol 2021;85(04):975-976[published online ahead of print, 2021 Jul 21]

29 Gohary YM, Abdel Fattah DS. Detection of tumor necrosis factoralpha in nonlesional tissues of alopecia areata patients: a prove for a systemic disease. Int J Trichology 2017;9(04):154-159

30 Kanwar AJ, Narang T. Anagen effluvium. Indian J Dermatol Venereol Leprol 2013;79(05):604-612

31 Gianotti R, Zerbi P, Dodiuk-Gad RP. Clinical and histopathological study of skin dermatoses in patients affected by COVID-19 infection in the Northern part of Italy. J Dermatol Sci 2020;98(02): 141-143

32 Magro C, Mulvey JJ, Berlin D, et al. Complement associated microvascular injury and thrombosis in the pathogenesis of severe COVID-19 infection: a report of five cases. Transl Res 2020;220:1-13

33 Watras MM, Patel JP, Arya R. Traditional anticoagulants and hair loss: a role for direct oral anticoagulants? A review of the Literature. Drugs Real World Outcomes 2016;3(01):1-6

34 Vaughn VM, Yost M, Abshire C, et al. Trends in venous thromboembolism anticoagulation in patients hospitalized with COVID19. JAMA Netw Open 2021;4(06):e2111788

35 Luo M, Guo L, Yu M, Jiang W, Wang H. The psychological and mental impact of coronavirus disease 2019 (COVID-19) on medical staff and general public - a systematic review and metaanalysis. Psychiatry Res 2020;291:113190

36 Rajkumar RP. COVID-19 and mental health: a review of the existing literature. Asian J Psychiatr 2020;52:102066

37 Brooks SK, Webster RK, Smith LE, et al. The psychological impact of quarantine and how to reduce it: rapid review of the evidence. Lancet 2020;395(10227)912-920
38 Nguyen $\mathrm{H}$, Tran N, et al. Perceived stress of quarantine and isolation during COVID-19 pandemic: a global survey. Frontiers in Psychiatry, Volume 2021;12:651

39 Botchkarev VA. Stress and the hair follicle: exploring the connections. Am J Pathol 2003;162(03):709-712

40 Chu B, Marwaha K, Sanvictores T, et al. Physiology, Stress Reaction. [Updated 2021 Jun 8]. In: StatPearls [Internet]. Treasure Island (FL): StatPearls Publishing; 2021 Jan-. Available from: https://www.ncbi.nlm.nih.gov/books/NBK541120/

41 Peters EMJ, Müller Y, Snaga W, et al. Hair and stress: a pilot study of hair and cytokine balance alteration in healthy young women under major exam stress. PLoS One 2017;12(04):e0175904

42 Hadshiew IM, Foitzik K, Arck PC, Paus R. Burden of hair loss: stress and the underestimated psychosocial impact of telogen effluvium and androgenetic alopecia. J Invest Dermatol 2004;123(03): 455-457

43 FIvenson D. COVID-19: association with rapidly progressive forms of alopecia areata. Int J Dermatol 2021;60(01):127

44 Malkud S. Telogen effluvium: a review. J Clin Diagn Res 2015;9 (09):WE01-WE03

45 Grover C, Khurana A. Telogen effluvium. Indian J Dermatol Venereol Leprol 2013;79(05):591-603

46 Rebora A. Telogen effluvium: a comprehensive review. Clin Cosmet Investig Dermatol 2019;12:583-590

47 McDonald KA, Shelley AJ, Colantonio S, Beecker J. Hair pull test: evidence-based update and revision of guidelines. J Am Acad Dermatol 2017;76(03):472-477

48 Jain N, Doshi B, Khopkar U. Trichoscopy in alopecias: diagnosis simplified. Int J Trichology 2013;5(04):170-178. Doi: 10.4103/0974-7753.130385 